\title{
NATURAL SELECTION FOR ENVIRONMENTALLY INDUCED PHENOTYPES IN TADPOLES
}

\author{
Josh Van Buskirk, ${ }^{1}$ S. Andy McCollum, and Earl E. Werner \\ Department of Biology, University of Michigan, Ann Arbor, Michigan 48109
}

\begin{abstract}
Models suggest that phenotypic plasticity is maintained in situations where the optimal phenotype differs through time or space, so that selection acts in different directions in different environments. Some empirical work supports the general premise of this prediction because phenotypes induced by a particular environment sometimes perform better than other phenotypes when tested in that environment. We have extended these results by estimating the targets of selection in Pseudacris triseriata tadpoles in environments without predators and with larval Anax dragonflies. Tadpoles displayed significant behavioral and morphological plasticity when reared in the presence and absence of nonlethal dragonflies for 32 days in cattle tanks. We measured selection in the absence of free predators by regressing growth and survival in the tanks against activity and several measures of tail and body shape. We measured selection in the presence of predators by exposing groups of 10 tadpoles to Anax in overnight predation trials and regressing the average phenotype of survivors against the number of tadpoles killed. Selection in the two environments acted in opposite directions on both tail and body shape, although the affected fitness components were different. In the presence of Anax, tadpoles with shallow and narrow body, deep tail fin, and wide tail muscle survived best. In the absence of free predators, tadpoles with narrow tail muscle grew significantly faster, and those with shallow tail fin and deep body grew somewhat faster. Activity was unrelated to survival or growth in either environment. Developmental plasticity in tail shape closely paralleled selection, because tail fin depth increased after long-term exposure to Anax and tail muscle width tended to increase. In contrast, there was no plasticity in body shape in spite of strong selection for decreasing body depth. Thus, when confronted with a dragonfly predator, $P$. triseriata tadpoles adjusted their tail shape (but not body shape) almost exactly in the direction of selection imposed by Anax. These results suggest that phenotypic plasticity in some morphological traits, such as tail depth and tail muscle width, has evolved under intermittent selection by dragonflies. Other traits that undergo selection by dragonflies, such as body morphology, appear developmentally rigid, perhaps because of historically strong opposing selection in nature or other constraints.
\end{abstract}

Key words.-Antipredator behavior, inducible defense, phenotypic plasticity, predation, Pseudacris triseriata, selection, trade-off.

Received October 21, 1996. Accepted June 30, 1997.

Environmental heterogeneity in space or time presents a fundamental challenge for organisms. In the absence of environmental heterogeneity the phenotype conferring maximum fitness is expected to evolve, subject to existing genetic or functional constraints. However, if the phenotype conferring maximum fitness changes in some way as the environment changes, no single optimal phenotype exists. There are several possible evolutionary responses to environmental heterogeneity, including genetic polymorphism, ecological generalization, habitat selection, and phenotypic plasticity. Determining the conditions that favor one or another of these alternatives has been a topic of long-standing interest (Levene 1953; Lewontin 1957; Hedrick 1986; Futuyma and Moreno 1988; Thompson 1994). Theory suggests that phenotypic plasticity, defined as the ability of a genotype to produce different phenotypes in different environments, should be favored when a reliable cue indicates the state of the environment, dispersal is restricted, and there is a trade-off in the fitness of phenotypes in different environments (Lively 1986a; Dodson 1989; Moran 1992). In fact, many plants and animals display apparently adaptive plasticity, which implies that these conditions must often be met in nature (Schlichting 1986; Dodson 1989).

Measurements of the performance of different phenotypes or genotypes across environments sometimes indicate the existence of fitness trade-offs (e.g., Cook and Johnson 1968;

I Present address: Institute of Zoology, University of Zürich, $\mathrm{CH}$ 8057 Zürich, Switzerland; E-mail: jvb@zool.unizh.ch.
Via 1984; Harvell 1986; Lively 1986b; Karban and Meyers 1989; McCollum and Van Buskirk 1996). Typically, the fitnesses of different phenotypes are assessed in multiple environments, and the results indicate a trade-off if the phenotype with the highest fitness in one environment performs less well in other environments. Such evidence for trade-offs illustrates that the phenotypic optima differ among environments, but does not demonstrate what the optima are for each environment. Models for the evolution of plasticity require knowledge of the locations of divergent optimal phenotypes, or estimates of the fitness functions within each environment, in order to predict the configuration of reaction norms conferring high fitness (Via and Lande 1985; Gomulkiewicz and Kirkpatrick 1992; Via 1994). Furthermore, direct measurements of selection in the separate environments are necessary to determine if the same traits that exhibit plasticity are currently under divergent selection. Otherwise, we cannot rule out the possibility that plastic traits are exhibiting spurious variation as environmental conditions change, or that traits vary with the environment only because they are connected to unknown adaptively plastic traits via pleiotropy or a genetic correlation. Thus, direct measurement of selection can help test whether plasticity is an adaptation currently maintained by variability in the environment.

We studied plasticity and selection in tadpoles of the chorus frog (Hylidae: Pseudacris triseriata) to test whether selection acts in conflicting directions in environments characterized by different predation regimes. Most of the conditions favoring plasticity are present in the small ponds inhabited by 
Pseudacris tadpoles: waterborne chemical signals provide an indication of environmental conditions, and dispersal is restricted by the extent of the pond. Previously, we have described morphological plasticity in Pseudacris and Hyla tadpoles occurring in environments with and without larval dragonfly predators (Smith and Van Buskirk 1995; McCollum and Van Buskirk 1996; McCollum and Leimberger 1997), and we demonstrated a trade-off in the performance of the two phenotypes in the presence and absence of dragonflies (McCollum and Van Buskirk 1996). However, we do not yet know how selection acts on tadpole phenotypes in the different environments. We addressed this problem by comparing the flexible morphological and behavioral responses to two distinct environments with the directions of selection acting on plastic traits of tadpoles in those two environments. The results illustrate that natural selection on tail shape is imposed in nearly opposite directions in the two environments, and that morphological plasticity is closely aligned with selection. Our results therefore strengthen the case that plasticity in anuran tail shape is maintained by spatially or temporally fluctuating selection acting directly on tail shape.

\section{Methods}

We studied the relationship between phenotypic plasticity and selection in tadpoles of the chorus frog, Pseudacris triseriata. Previous research has established that this species has flexible behavioral and morphological responses to predators (Skelly 1995; Smith and Van Buskirk 1995). Eggs were collected in early April 1995 from a large population of $P$. triseriata in Tinkle's Marsh on the E. S. George Reserve of the University of Michigan in southeastern Michigan. At least 50 females contributed eggs to the experiments. We maintained eggs in $300-\mathrm{L}$ outdoor wading pools until the experiment began, 4-7 days after the tadpoles hatched.

\section{Plasticity Experiment}

We measured morphological plasticity in $P$. triseriata exposed to the presence or absence of nonlethal dragonfly predators. The experiment was conducted outdoors in artificial ponds for one month. Ponds were established in black plastic stock tanks filled with well water to a depth of $41 \mathrm{~cm}(1100$ $\mathrm{L}$ volume, $2.6 \mathrm{~m}^{2}$ surface area). Each tank was stocked with three well-mixed collections of zooplankton and phytoplankton taken from nearby ponds; $0.5 \mathrm{~kg}$ oak leaf litter and $25 \mathrm{~g}$ commercial rabbit chow were added to provide nutrients to support the foodweb. We covered the tanks with screen lids to prevent unwanted colonization by insects and amphibians, and arranged them $0.8 \mathrm{~m}$ apart in a hexagonal array in an open field. Experimental blocks were grouped according to spatial proximity within this array and the date on which tadpoles were introduced.

We manipulated the presence or absence of caged Anax dragonfly larvae (mixture of $A$. junius and A. longipes, Odonata: Aeshnidae) in the tanks, and replicated the two treatments eight times. Each tank contained three floating cages constructed of plastic drain pipe $(11 \mathrm{~cm}$ diameter $\times 12 \mathrm{~cm}$ length) capped with fiberglass windowscreen; in the predatorfree treatment the cages were empty, and in the predator treatment we confined a single late-instar Anax larva within each cage. Dragonflies were fed 9-12 $P$. triseriata tadpoles every other day, the number depending on the size of the tadpoles. We have found that Anax larvae confined in such cages effectively present the perception of predation risk (McCollum and Van Buskirk 1996; Werner and Anholt 1996).

The eight experimental blocks were initiated between 25 April and 2 May 1995, when we introduced 250 tadpoles to each tank (density of $95 / \mathrm{m}^{2}$ ). We preserved a sample of 20 tadpoles for morphological measurements from each tank after 32 days, and drained seven of the eight blocks at that time to count and weigh the survivors. One block was left filled as part of a separate investigation.

We measured behavioral responses of $P$. triseriata to caged dragonflies by observing tadpoles in the tanks during midafternoon on sunny days about halfway through the experiment. For five haphazardly chosen tadpoles in each tank, we recorded the time spent resting, swimming, and feeding. Focal tadpoles were watched for $1 \mathrm{~min}$ each by an observer sitting beside the tank with a laptop computer programmed to convert sequences of keystrokes into the total time spent in each activity.

We measured morphology of the preserved tadpoles using a dissecting scope and camera connected to a computer installed with Bioscan Optimas image analysis software (Optimus Corp., Bothell, Washington). With the tadpole on its side, we digitized the length and depth of the body and the maximum depth of the tail fin and tail muscle at the base of the tail. Viewing the tadpole from the top, we digitized body width and maximum width of the tail muscle. We performed a principal component analysis on the covariance matrix constructed from these six measures and the wet mass of the preserved tadpole, and kept the first component (85\% of the total variance) as a measure of overall body size for subsequent analyses.

Specimens from the plasticity experiment were digitized 1-2 months after preservation, whereas those from the selection experiment (see below) were digitized six months later. As a result, formalin-induced shrinkage had progressed to different degrees in the two sets of tadpoles. We adjusted measurements for shrinkage by first regressing traits against one another and then estimating the extent of shrinkage in the selection experiment (relative to the plasticity experiment) for each trait.

We tested the significance of plastic responses to predators by comparing the morphology of tadpoles from the no-predator and caged-predator tanks after 32 days, using mixedmodel multivariate ANOVA (predator environment was treated as a fixed effect and tested over its interaction with block). Tank means were the unit of analysis, estimated from the 20 tadpoles collected from each tank. We focused on three measures of tail shape and two measures of body shape. These five measures were the residuals of log-transformed tail fin depth, tail muscle depth, tail muscle width, body depth, and body width after regression against the first principal component. $\mathrm{PC} 1$ is a composite measure of size, and the residuals of tail and body measures on $\mathrm{PCl}$ gave us size-corrected measures of shape that are known to vary across predator environments in $P$. triseriata and other anurans (Smith and Van Buskirk 1995; McCollum and Van Buskirk 1996). Anal$y$ sis of activity employed mixed-model ANOVA in which the 
response was the average $(\arcsin \sqrt{\mathrm{p}}$-transformed) proportion of time tadpoles were active (feeding and swimming) in each tank.

\section{Selection in the Absence of Anax}

We estimated natural selection in the absence of free predators by examining the relationship between phenotype and performance in the plasticity experiment. Tadpoles in cattle tanks experienced no mortality from predators, so correlations between phenotype and growth or survival reflect natural selection in the absence of predators. We used multiple regression to ask whether two estimates of performance (survival and mass after 32 days) were related to predator treatment, activity level, and the five morphological measures at the end of the experiment. Thus, our analysis distinguished between the overall survival or growth cost of responding to predators (the effect of predator treatment), and selection acting on particular traits (effects of morphology and behavior). The analysis was performed on tank means because two of the variables (survival and activity) were collective responses of populations which could not be associated with individuals.

Our fitness measures for this analysis included both survival and growth rate. We expected selection in predator-free environments to be more clearly reflected in growth than survival because possessing inferior traits when predators are absent is more likely to result in individual growth or developmental costs than in death (Harvell 1990). Survival is clearly a good correlate of fitness (Endler 1986) and larval growth is also justifiable for anurans because reduced growth is associated with lower survival to metamorphosis (Wilbur 1984), and is further related to fitness through its impact on adult survival or age and size at reproduction (Smith 1987; Semlitsch et al. 1988; Berven 1990; Scott 1994).

\section{Selection in the Presence of Anax: Predation Trials}

We estimated natural selection on $P$. triseriata morphology in environments containing dragonflies by exposing tadpoles to free-ranging Anax and determining whether the predators killed a nonrandom sample of individuals in short-term predation trials. Selection on behavior was estimated by measuring activity just prior to beginning the predation trials, and determining whether survival during the trial was related to the average activity level of the tadpoles. We conducted predation trials in opaque plastic tubs $(55 \mathrm{~cm} \times 42 \mathrm{~cm}$, containing $45 \mathrm{~L}$ water), covered with screen lids and placed outdoors in an open field. To provide habitat structure we added a standard quantity of plastic rope to each tub, arranged into six clumps along a wooden dowel held in place with a rock.

The trials were performed on tadpoles from the caged-Anax treatment of the plasticity experiment because this represents the natural situation in which tadpoles that undergo predation in nature also exhibit the predator-induced phenotype. At the end of the plasticity experiment, we collected 120 tadpoles from each tank and divided them haphazardly into 12 sets of 10 tadpoles: four of these sets were chosen at random and placed into tubs with no predators, and eight sets were placed into tubs with one Anax. This was repeated for four replicate
caged-Anax tanks, making a total of 16 tubs with no predator and 32 tubs with Anax. We fed the dragonflies a single tadpole and confined them in small cages for the first $24 \mathrm{~h}$ to allow the tadpoles to become aware of the proximity of predators.

We observed behavior of the tadpoles the day after they were introduced to the tubs, between 11:00-15:00 h. An observer moved slowly among the tubs and scored the number of visible tadpoles that were swimming, feeding, or inactive. The tadpoles did not respond to the presence of the observer so long as movement was gradual and observations were made while still at some distance from the tub. We repeated this process three times at intervals of $>45 \mathrm{~min}$ and averaged the results to yield an estimate of activity for each tub.

After completing the behavioral observations, we released the dragonflies from their cages and allowed the predation trials to run overnight. We collected all surviving tadpoles the following morning and preserved them in $10 \%$ formalin for later morphometric analysis.

Ideally, natural selection imposed by predators is measured by associating survival with individual phenotypes (Endler 1986; Brodie et al. 1995). This was not possible in our predation trials because we were unable to mark individuals. Instead, we estimated selection on morphology in the presence of dragonflies by regressing the mean of each morphological trait within a tub against the number of tadpoles surviving in the tub. When no predators were present the number surviving was always 10 , and these tubs served as estimates of the phenotype prior to selection. If Anax killed tadpoles nonrandomly with respect to phenotype, we expected to see the average phenotype of the population deviate from its initial value as more tadpoles were killed. We tested for selection on behavior in the presence of Anax by regressing the proportion of tadpoles active just before releasing the dragonfly against the number killed during the trial; tubs without predators were not included in this analysis because there was always perfect survival regardless of the tadpoles' activity level.

\section{Comparison of Selection and Plasticity}

One objective of these experiments was to determine if natural selection favored different phenotypes in the two environments, as predicted by the hypothesis that plasticity is maintained by divergent selection. We addressed this question by comparing the direction of phenotypic change due to plasticity with the directions of change due to selection. The comparison involved three kinds of datasets:

(1) Plasticity: differences in morphology resulting from exposure to Anax and no-Anax treatments in the 32-day plasticity experiment in cattle tanks, expressed in units of standard deviation of the trait in the no-predator tanks.

(2) Selection in the absence of predators: differences in morphology of tadpoles between cattle tanks having either high or low performance (survival or growth). The direction and magnitude of selection for each trait (in SD units) was given by the slope of its regression against performance. This slope represents the change in average phenotype associated with a unit change in performance.

(3) Selection by Anax: differences in morphology arising from nonrandom predation by dragonflies in predation trials. 
TABLE 1. Univariate analyses on morphological and behavioral plasticity of Pseudacris triseriata tadpoles. Morphological measurements were made after 32 days in the presence or absence of Anax dragonflies, and were corrected for body size (residuals after regression on PC1). Activity (proportion of time spent swimming or feeding) was estimated halfway through the experiment. Entries in the table show $F$ (above) and $P$-value (below). The error is the block-by-treatment interaction. Tail fin depth increased, and activity decreased, significantly in the presence of dragonflies.

\begin{tabular}{llccccc}
\hline \hline \multirow{2}{*}{ Source (df) } & \multicolumn{5}{c}{ Response } \\
\cline { 2 - 6 } & Body width & Body depth & Muscle width & Muscle depth & Tail fin depth & Activity \\
\hline Block $(7,7)$ & 6.60 & 7.87 & 3.83 & 2.49 & 2.32 & 3.63 \\
& 0.0118 & 0.0071 & 0.0486 & 0.1261 & 0.1449 & 0.0552 \\
Predator treatment $(1,7)$ & 0.12 & 0.04 & 1.26 & 2.79 & 7.74 & 18.20 \\
& 0.7417 & 0.8487 & 0.2992 & 0.1390 & 0.0272 & 0.0037 \\
\hline
\end{tabular}

The direction and magnitude of selection was given by the slope of the regression of phenotype (in SD units) on the number of tadpoles killed by Anax. This slope corresponds to the change in the average phenotype of survivors in the population resulting from the death of one tadpole. The relationship is expected to be slightly sigmoidal if the predator imposes perfect truncation selection (Falconer and Mackay 1996), but our results appeared roughly linear (see Results).

We employed a nonparametric comparison of selection and plasticity because the three datasets were not independent and not directly comparable. For example, tadpoles used in the Anax-selection experiment were drawn from one of the treatments in the plasticity experiment and estimates of selection in the absence of predators also used results from the plasticity experiment. Also, the two estimates of selection were conducted under different time frames and using different measures of fitness, making problematic any direct comparison of the magnitudes of selection between the two environments. We used the change in mean phenotype due to plasticity in each trait to predict the direction of selection within each environment, and then used a binomial test to determine if selection and plasticity were congruent, as predicted if selection acts to maintain plasticity.

\section{RESULTS}

\section{Phenotypic Plasticity}

There were differences in behavior and tail shape between Pseudacris triseriata in the Anax and no-Anax environments,

TABLE 2. Covariance analysis on the average log-transformed final mass of Pseudacris triseriata in the plasticity experiment. Activity is the proportion of time spent feeding and swimming; the measures of body shape are residuals after regression on body size. In these tanks with no free-ranging predators, populations of tadpoles with relatively narrow tail muscle grew to a large final size and were therefore favored by selection. There was no selection on behavior or any other morphological trait.

\begin{tabular}{lrcccc}
\hline \hline \multicolumn{1}{c}{ Source } & df & SS & MS & $F$ & $P$ \\
\hline Model & 7 & $\mathbf{1 . 8 3 7}$ & 0.262 & 5.78 & 0.0242 \\
Error & 6 & 0.273 & 0.045 & & \\
Total & 13 & 2.110 & & & \\
Predator treatment & 1 & 0.001 & 0.001 & 0.03 & 0.8627 \\
Activity & 1 & $\mathbf{0 . 0 2 2}$ & 0.022 & 0.047 & 0.5169 \\
Body depth & 1 & $\mathbf{0 . 0 4 8}$ & 0.048 & 1.06 & 0.3429 \\
Body width & 1 & $\mathbf{0 . 0 6 6}$ & 0.066 & 1.45 & 0.2735 \\
Tail muscle width & 1 & $\mathbf{0 . 7 6 9}$ & 0.769 & 16.92 & 0.0063 \\
Tail muscle depth & 1 & 0.022 & 0.022 & 0.049 & 0.5090 \\
Tail fin depth & 1 & $\mathbf{0 . 0 3 2}$ & 0.032 & 0.71 & 0.4329 \\
\hline
\end{tabular}

reflecting significant phenotypic plasticity (Table 1 ). Tadpoles in the presence of caged predators spent less time actively swimming or feeding, and more time resting, than tadpoles in the no-predator tanks (mean \pm SE: $24.5 \pm 2.9 \%$ of time active in Anax treatment and $36.4 \pm 3.1 \%$ active in noAnax treatment). Multivariate comparisons between the two predator environments revealed no difference in size-specific body depth and width (Wilks $F_{2,6}=0.05, P=0.95$ ), but a nearly significant effect on the three measures of tail shape between the two environments (Wilks $F_{3,5}=4.55, P=$ 0.068). All three tail measurements (relative tail muscle depth, muscle width, and tail fin depth) increased in the presence of Anax and tail fin depth showed a significant change in univariate ANOVA (Table 1). Significant block effects probably stemmed from differences in timing, spatial position, and genetic composition.

\section{Estimates of Natural Selection}

In the absence of predation there was significant selection against tadpoles showing traits characteristic of high-predation environments. The presence or absence of caged Anax did not influence final mass or survival of tadpoles in cattle tanks, indicating no measurable cost of responding to predators (mass: $F_{1,6}=0.83, P=0.40$; survival: $F_{1,6}=2.10, P$ $=0.20$ ). However, mass was significantly higher in tanks that had tadpoles with narrow tail muscle (Table 2, Fig. 1). This analysis was performed on population means, but we obtained similar results from an analysis on the final mass of individual tadpoles that included tanks in the model (but necessarily did not include activity, since marked individuals were not observed). This implies that individuals with wide tail muscles grew slowly within tanks, regardless of treatment. No other traits were correlated with final mass and the corresponding analyses for survival were not significant. We interpret this as evidence for selection favoring tadpoles with relatively narrow tail musculature in environments without predators.

Feeding Anax imposed significant selection on $P$. triseriata tail and body shape (Fig. 2). We determined which traits experienced selection by comparing the morphology of selected and unselected groups of tadpoles in the predation trials. When a population of tadpoles suffered high mortality the surviving individuals were morphologically distinct from unselected tadpoles (Wilks' $F_{5,37}=5.39, P=0.0008$, effect of number killed in a MANOVA on all five measures of body and tail shape). Univariate tests showed that individuals re- 


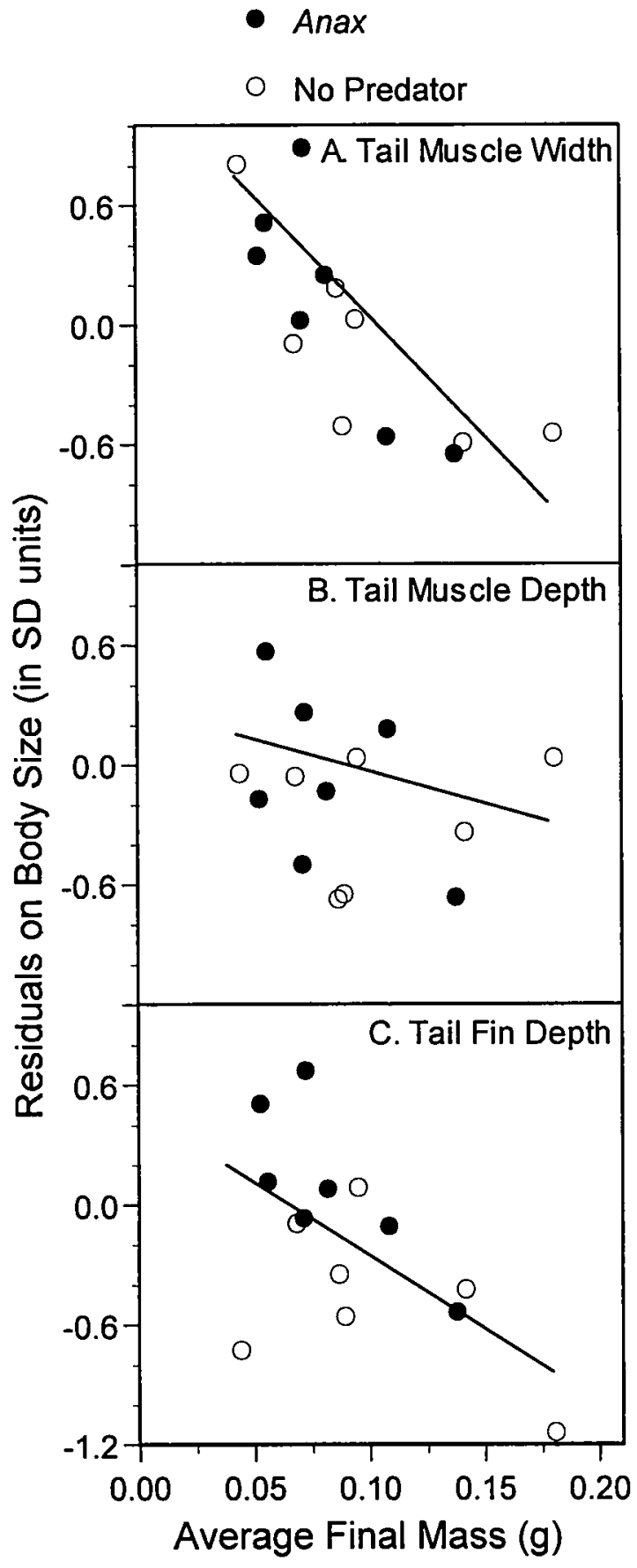

FIG. 1. Selection on morphology of Pseudacris triseriata tadpoles in the absence of predation, depicted as relationships between tail shape and mass at the end of the experiment (a correlate of fitness). Morphological measures were corrected for body size by first regressing on body size (PC1), and are expressed in units of SD among individuals within tanks. The negative relationships between all three measures and final mass reflect selection favoring tadpoles with relatively narrow tail muscles and shallow tail fins when predators are not killing tadpoles.

maining alive at the end of the predation trials had narrower and shallower bodies, wider tail muscles, and deeper tail fins than did tadpoles prior to selection (Fig. 2), but there was no effect on tail muscle depth. This indicates that Anax killed tadpoles nonrandomly with respect to body volume, tail muscle width, and tail fin depth, but not tail muscle depth.

Anax did not impose selection on tadpole behavior. There was no relationship between survival during the predation trial and the proportion of tadpoles actively feeding or swimming just before the dragonflies were released $\left(F_{1,25}=0.10\right.$, $P=0.749$ ). Populations composed of relatively inactive tadpoles did not survive better in the presence of predators, as expected if activity increases vulnerability to predation.

\section{Comparison of Plasticity and Selection}

Shifts in average phenotype due to selection in the presence and absence of predators were in opposite directions for all five measures of tail and body shape, as expected if selection acts to maintain plasticity (Fig. $3 ; P=0.001$, binomial test). Relative body depth and width both decreased slightly after long-term exposure to predators, while selection by Anax favored smaller body measures and selection in the no-predator environment was in the opposite direction. Similarly, the three tail shape measures increased after exposure to Anax, while selection by dragonfly predation caused an increase in all three measures and selection in the no-predator environment favored decreases in all three (Fig. 3).

Visual inspection of the results illustrates that selection and plasticity were generally congruent for tail shape but not for body shape (Fig. 4). There was strong selection on body shape in the presence of Anax but no significant selection in the no-predator environment, and there was no developmental plasticity in body shape (Fig. 4A). In contrast, selection on tail shape in the two environments was highly significant and acted in opposite directions, and plasticity was more or less aligned with selection (Fig. 4B). In detail, the directions of selection and plasticity were not identical, because the strongest plastic response to dragonflies was in tail fin depth, whereas selection acted more strongly on tail muscle width. However, all three measures of tail shape showed some degree of plasticity and selection, so that the net effect was a high degree of congruence between plasticity and selection on tail shape.

\section{Discussion}

This study clarifies some of the evolutionary mechanisms responsible for maintenance of flexible responses to predators in anuran larvae. Natural selection on body and tail shape in Pseudacris triseriata tadpoles occurred in the presence and absence of predators, and the selection regime in environments lacking free predators was strongly divergent from that in ponds with dragonflies preying on tadpoles. Our estimates of selection extend results with another anuran species showing that two phenotypes experience a performance trade-off across environments (McCollum and Van Buskirk 1996). In the present study, the agreement between the targets of selection and the traits showing plasticity in the tail strengthens the evidence that plasticity is maintained by divergent selection imposed by environments with and without predators. The incongruence between selection and plasticity in traits relating to body shape may reflect a design constraint that prevents tadpoles from facultatively altering body shape. 

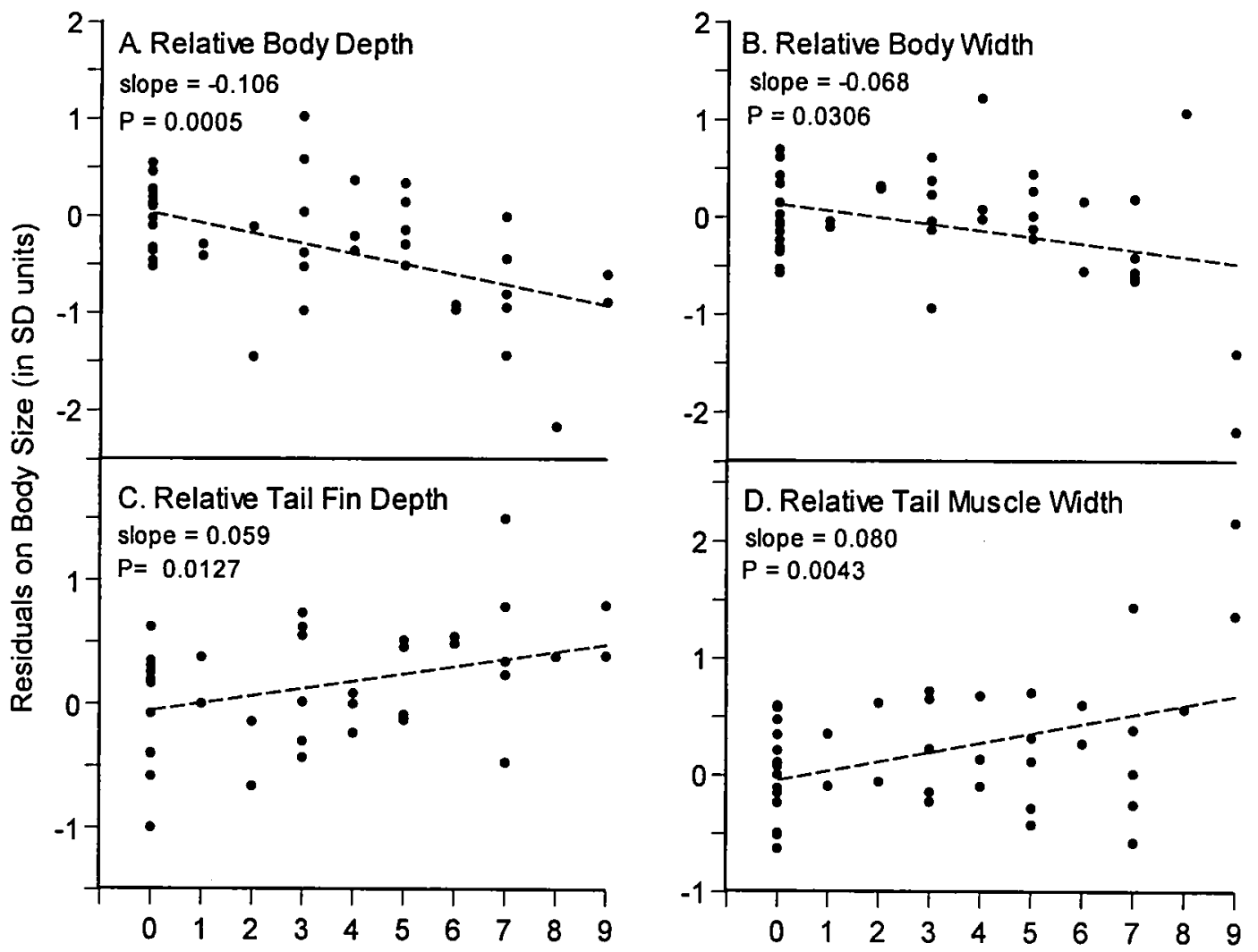

Number Killed by Predators

FIG. 2. Selection by Anax dragonflies on morphology of Pseudacris triseriata tadpoles in short-term predation trials. All trials began with 10 tadpoles. Each point shows the average measurement for all survivors within an experimental unit, corrected for body size and expressed in units of SD of the trait in the population prior to selection. Individuals that survived the trials differed from the initial samples of tadpoles, demonstrating that Anax imposed selection on these four measures of body and tail shape. The magnitude of selection is measured by the slope, which gives the average phenotypic change (in SD units) for each tadpole killed.

\section{Selection in the Anax Environment}

Selection imposed by feeding dragonflies in the predation trials could arise either from an unknown preference by the predators for specific tadpole traits, or from variation in escape capability among tadpoles possessing different traits. Foraging predators often display preferences for certain food types (Begon et al. 1996), and if the predator's preference is correlated with phenotypic variation within the prey population, the process would produce selection such as we observed. So far as we are aware, though, Anax does not show a preference for tadpoles of a particular shape and individuals of both phenotypes are equally palatable.

It seems probable that selection during predation trials reflected differences among individuals in traits that enhance ability to escape or avoid detection by predators. Anax tended to kill tadpoles that had deep, wide bodies, shallow tail fins, and narrow tail muscles. Although the functional consequences of these traits are uncertain, our results suggest that they are probably related to a tadpole's ability to accelerate suddenly and swim rapidly, which is critical for predator escape in other anurans (Feder 1983; Watkins 1996). Our predation trials probably were not sensitive to selection acting on predator-avoidance traits, such as habitat selection and crypsis, because of the relatively small size of the experimental units.

The relationship between tail fin depth and ability to escape predators may be widespread in larval anurans. Tadpoles of several species develop deep tail fins when exposed to predators (Smith and Van Buskirk 1995; McCollum and Van Buskirk 1996; Relyea and Werner, unpubl.), and the present study demonstrates that tail fin depth is under selection to increase in the presence of dragonflies. The high point of the fin may be the most effective part of the tail for generating thrust in tadpoles (Wassersug and Hoff 1985), and McCollum and Leimberger (1997) discovered a positive relationship between maximum tail fin depth and swimming speed. Yet in fish, only the depth of the trailing edge is related to propulsive force (Webb 1984) and increasing fin depth anterior to the trailing edge in chaetognathes does not increase propulsion (Jordan 1992). Thus, although the association of tail fin depth with predator escape implies a functional connection, biomechanical models of swimming in fish, anuran larvae, and invertebrates encourage us to exercise caution in interpreting functional consequences of morphological variation.

Tadpoles do not use muscles in the tapered portion of the tail for generating thrust in steady swimming (Blight 1976, 


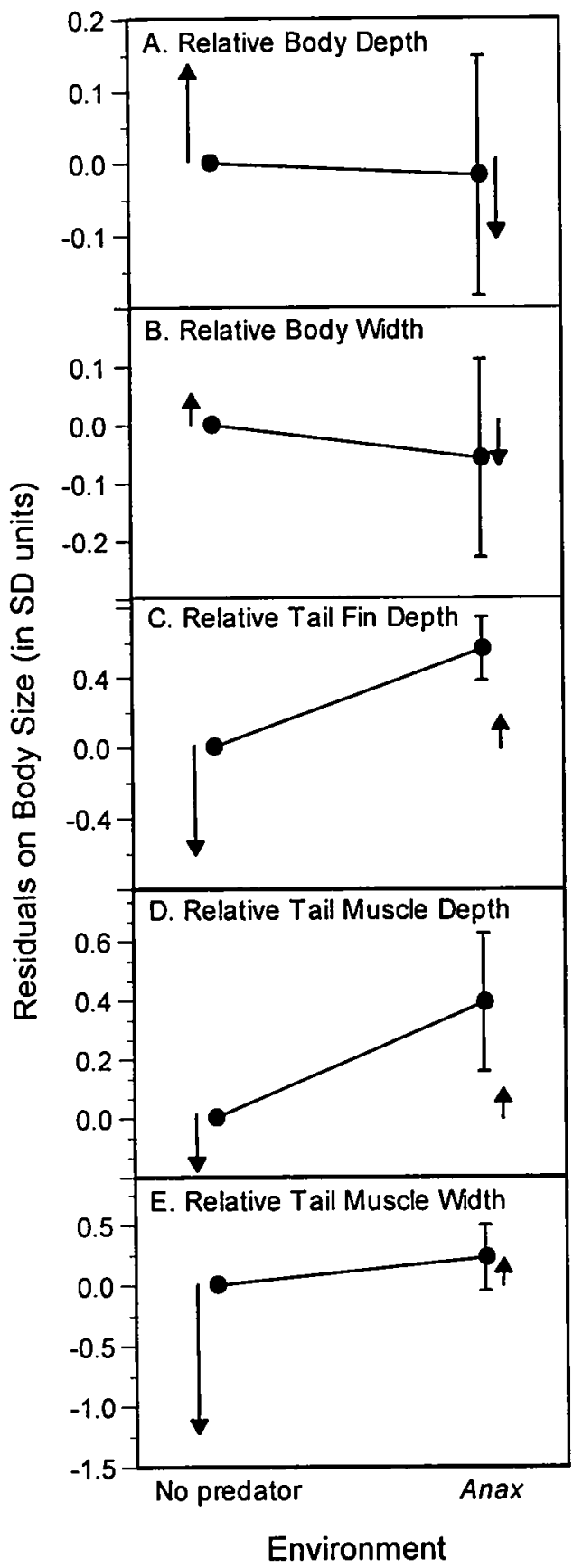

FIG. 3. Comparison of phenotypic plasticity in five measures of Pseudacris triseriata body and tail shape with natural selection on the same traits. The sloped line represents plasticity after 32 days in the presence or absence of caged Anax; the phenotypic value in the predator-free environment is set to zero and therefore has no error bars. The phenotypic change is expressed in SD units (mean $\pm 1 \mathrm{SE}$ ). Vertical arrows depict selection in the two environments, and are arbitrarily anchored at zero. Selection in the presence of Anax was measured by survival in overnight predation trials; the length of the arrow corresponds to the slope of the relationship between phenotype (in SD units) and mortality (see Fig. 2). Selection in the no-predator environment was measured by growth rate in cattle tanks; the length of the arrow corresponds to the slope of the relationship between phenotype (in SD units) and final mass in grams (see Fig. 1 ). Selection favored tadpoles with opposite combinations of traits in the two environments.
1977), but these muscles are employed during sudden acceleration and turning (Wassersug and Hoff 1985; Wassersug 1989). Thus, our measures of tail muscle depth and width may be functionally related to fast-start capability and predator escape, even if they do not reflect performance in sustained swimming. This interpretation certainly agrees with our observation that Anax was more likely to kill tadpoles with narrow tail muscles.

Selection on body width and depth indicates that tadpoles with relatively elongate bodies were better able to escape predation, perhaps because of their streamlined shape. This result suggests an alternative explanation for the negative relationship between tail size and body volume in tadpoles (Smith and Van Buskirk 1995; McCollum and Van Buskirk 1996; Relyea and Werner, unpubl.). Earlier explanations for this relationship focus on the functional trade-off between swimming performance, related to tail fin and musculature (Wassersug 1989; McCollum and Leimberger 1997), and food processing capability, related to the volume of the buccal chamber and viscera (Wassersug and Hoff 1979; Nodzenski et al. 1989). Our study found no selection for large relative body size in the absence of hunting Anax, as expected under a tail size/body volume trade-off. Instead, predators directly imposed selection for small body size, which could be sufficient to generate a negative relationship between relative tail depth and body volume.

Activity level was not under selection in the predation trials. We believe that activity is less important than morphology in escaping a predator's attack, although movement frequency and speed certainly affect initial detection by predators (Woodward 1983; Folsom and Collins 1984; AzevedoRamos at al. 1992; Werner and Anholt 1993; Skelly 1994; Anholt and Werner 1995). To some extent, our results may depend on the arenas in which the predation trials were conducted. If predator-prey encounters were bound to occur frequently in our structurally simple containers, then the relationship between activity and encounter rate might be irrelevant, and morphological traits conferring the ability to escape inevitable attacks would then become critical. Alternatively, the trials may have lasted long enough for the Anax to kill until they were satiated, in which case the number of tadpoles killed in a tub would depend on the motivation or hunger of the Anax rather than the average phenotype of their victims (which is how we measured activity). Thus, survival would be unrelated to the average activity of the tadpoles, although differences in behavior or morphology among tadpoles within a tub might still influence exactly which individuals survive. For these reasons, we remain uncertain about the relative contributions of behavior and morphology to predator-avoidance until experiments are conducted that can properly separate performance consequences of both modes of phenotypic variation.

\section{Selection in the Predator-Free Environment}

Selection in the absence of predation mortality was predominantly in the opposite direction from selection in the presence of free dragonflies. While Anax tended to kill tadpoles with relatively deep, wide bodies, shallow tail fins, and narrow and shallow tail muscles, tadpoles with these traits 

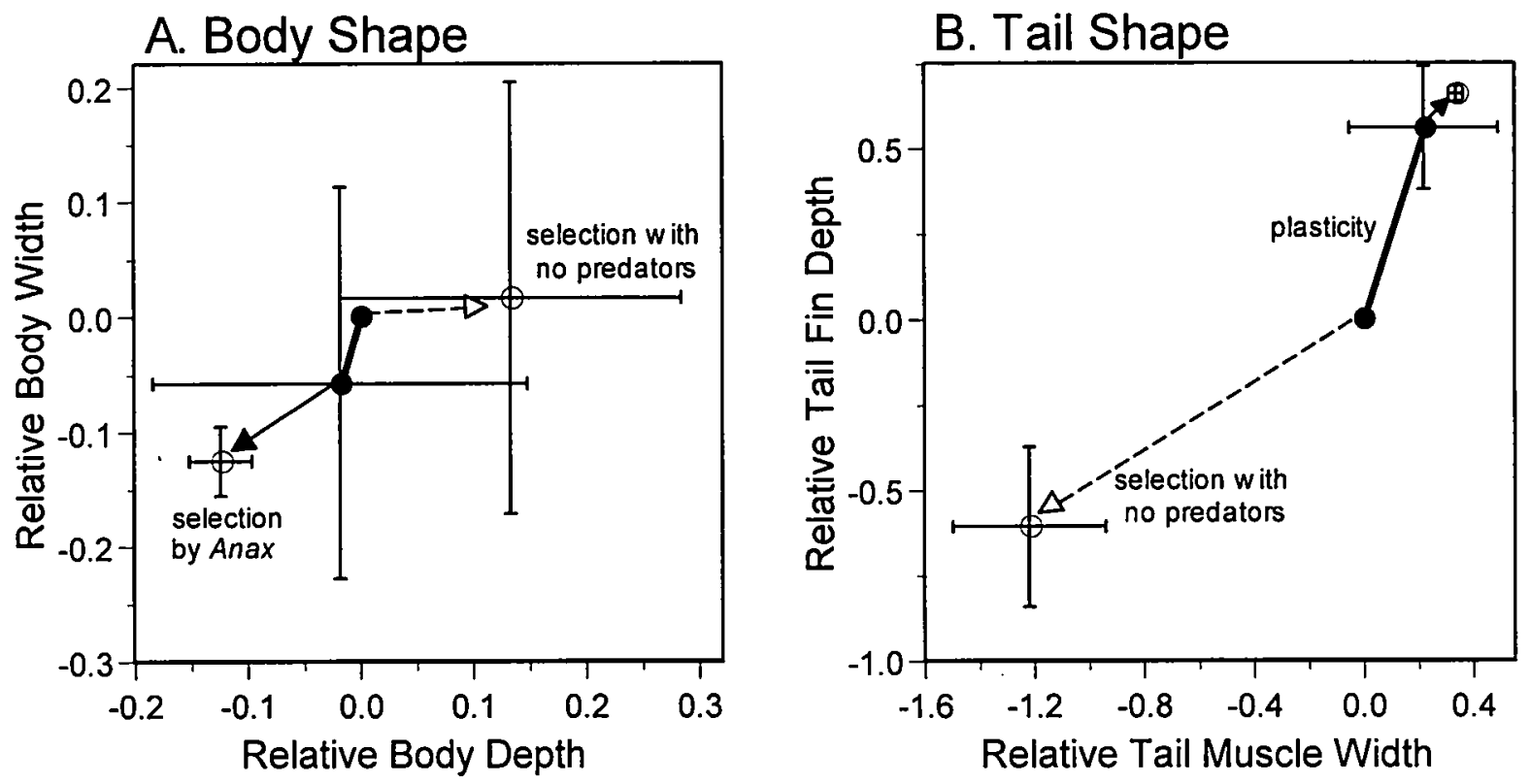

FIG. 4. Comparison of phenotypic plasticity and natural selection on body width and depth (A) and tail muscle width and tail fin depth (B) in Pseudacris triseriata tadpoles, depicted in bivariate phenotype space. The heavy solid line represents plasticity (mean \pm 1 SE); the phenotypic value in the no-predator treatment was set to zero and therefore has no error. The dashed arrows represent selection in the no-predator environment (mean $\pm 1 \mathrm{SE}$ ), and were arbitrarily anchored at the origin. The solid arrows, representing selection by Anax, originate at the final phenotypic value in the Anax environment since these tadpoles were used in the predation trials. For an explanation of data preparation see Figure 3.

tended to grow faster in the absence of predators. The directions of selection were opposite for all five of the traits that we measured (Fig. 3), although only tail muscle width was significantly correlated with performance in the no-predator environment. The overall pattern was consistent with the notion that traits useful for predator-escape are disfavored when predators are absent.

The reduced final mass of tadpoles with wide tail muscle cannot be interpreted as a simple growth cost of responding to the perception of predation risk, because the effect persists after first correcting for differences between predator treatments (Table 2). This means that, regardless of whether nonlethal dragonflies were present, tanks containing tadpoles with large average tail muscle width also had low average growth rate. A separate analysis on individual tadpoles, that included tanks in the model but did not include activity, also revealed a negative correlation between individual mass and muscle width, so selection acted against wide tail muscle within tanks regardless of treatment. Competition for food in the absence of predators is expected to generate natural selection for traits related to competitive ability and rapid growth rate (Wassersug and Hoff 1979; Nodzenski et al. 1989; Smith and Van Buskirk 1995). We therefore expected, but did not observe, selection for larger bodies and greater feeding activity in the predator-free environment. In both cases the trends were in the direction predicted, but were not significant.

Selection against tail muscle width in ponds without free predators may reflect a trade-off stemming from the expense of building or maintaining traits related to predator escape (Harvell 1990). If this were the case, though, we might expect to see a cost of responding to predators, measured as reduced growth or survival in tanks where tadpoles were exposed to caged Anax. We recorded no such cost, although an earlier study did find an apparent survival cost associated with the predator-induced phenotype (McCollum and Van Buskirk 1996). In fact, our results may reflect the growth cost of building wide tail muscle, but this cost is not included within the cost of the plastic response because tail muscle width shows relatively little plasticity (Fig. 3E).

\section{Selection for Phenotypic Plasticity}

Our results strengthen the case that phenotypic plasticity is maintained by selection favoring different phenotypes in different environments. They complement an extensive literature showing that phenotypes or genotypes with high fitness in one environment are poorly suited for other environments, presumably because selective optima vary among environments. Performance trade-offs have been especially well documented in plants and insect-plant interactions (Jain and Bradshaw 1966; Antonovics 1968; Berenbaum et al. 1986; Via 1991; Simms 1992; Futuyma et al. 1995; Schmitt et al. 1995), although good examples also exist for animals (Harvell 1986; Lively 1986b, 1986c; Spitze 1992; McCollum and Van Buskirk 1996). Many of these studies also show that plasticity is associated with the performance trade-off, supporting the hypothesis that plasticity is adaptive.

Our results estimating directionality of selection go beyond establishing a performance trade-off, because they help identify the precise traits that are favored in each environment. We cannot characterize the fitness functions in each environment without fitness estimates for a series of individuals having different phenotypes (e.g., Dudley and Schmitt 1996), 
but we can conclude that different combinations of traits confer high performance (in this case, survival or growth) in the two environments.

Why is plasticity nearly parallel to selection in the two environments? It seems likely that phenotypic variation exhibited by $P$. triseriat $a$ evolved in direct response to selective agents in the two environments. To make this case we need better data on the quantitative genetics of tadpole morphology, the distributions of various predator species in nature, and the behavioral mechanisms of prey capture for each predator. At present, for example, we do not know whether genetic correlations constrain the three measures of tail shape to vary along the same axis, ensuring that both selection and plasticity must follow the same trajectory. Selection by Anax may have acted directly on just a single trait, and other traits responded because they were genetically correlated with the target of selection. Of course this explanation would beg the question of why such a genetic correlation exists in the first place. Correlated selection on tail fin and muscles might generate a weak positive genetic correlation between them in the short term (Lande 1980, 1984), but in the long run such multiple-trait selection on tail shape would be expected to produce negative, rather than positive, genetic correlations (Sheridan and Barker 1974; Falconer and Mackay 1996). The genetic correlation hypothesis could be tested with studies of the quantitative genetics of morphology, along with selection experiments in which correlated components of the phenotype are experimentally disentangled.

Quantitative data on the exposure of $P$. triseriata to multiple predators in natural ponds might help evaluate whether phenotypic plasticity has evolved as a specific response to Anax or a generalized anti-predator response. The close agreement between selection and plasticity argues that plasticity was fairly specific, because it shows that selection by feeding Anax is capable of maintaining the plasticity that we measured. However, certain other insect predators capture tadpoles in much the same way as Anax and may apply selection for similar combinations of traits. A deepened tail, for example, may protect tadpoles against a variety of predators. Since $P$. triseriata is exposed to numerous predators in natural ponds (Skelly 1996), a specific response to each predator species would require tadpoles to be capable of distinguishing among predators and mounting the appropriate response when confronted with a particular species. We know too little about the hunting mechanisms employed by predators and the specificity of tadpole responses, to evaluate these possibilities at present. Preliminary data suggest that other anurans may show specific behavioral responses to different predators (R. A. Relyea, pers. comm.), which indicates that it is possible for tadpoles to distinguish among predators and respond accordingly.

The incongruence between selection and plasticity in relative body dimensions (Fig. $4 \mathrm{~A}$ ) implies that there may be a constraint on the ability of $P$. triseriata to reduce its body width and depth in the presence of predators. Hylid tadpoles in other studies have shown plasticity in relative body volume (Smith and Van Buskirk 1995; McCollum and Van Buskirk 1996), so our results may be related to the environment experienced by $P$. triseriata on our study area. Our data indicate that $P$. triseriata on the George Reserve has a longer, shal- lower, and narrower body than $P$. triseriata on Isle Royale (reanalyzed data from Smith and Van Buskirk 1995), and the George Reserve tadpoles also occur in predator-rich ponds more often than those on Isle Royale (compare Smith 1983 with Skelly 1996; J. Van Buskirk pers. obs.). Perhaps $P$. triseriata on the George Reserve has evolved an elongate body in response to frequent selection imposed by odonates and other predators, and is prevented from further plasticity by imperatives associated with feeding and digestion.

The anuran system joins many others for which there are trade-offs in the performance of multiple phenotypes across environments (references above). The general occurrence of such trade-offs in nature would be reassuring, since they constitute the foundation of virtually all models for the evolution of plasticity. It is difficult to imagine how plasticity could evolve or be mairtained if such trade-offs were not enforced at least some of the time. Furthermore, our results combine with a relatively few studies (Kingsolver 1995; Dudley 1996; Dudley and Schmitt 1996) to show how natural selection acts on plastic traits to favor the expression of divergent phenotypes in different environments. For a few model systems then, we are beginning to build a picture of how phenotypic plasticity is favored and maintained in environments characterized by spatial or temporal variability.

\section{ACKNOWLEDGMENTS}

We are grateful to K. Yurewicz for help with the fieldwork. For their comments on the manuscript we thank B. Anholt, A. Welch, and two anonymous reviewers. We were supported by NSF grant DEB-9408397.

\section{Literature Cited}

ANHOLT, B. R., AND E. E. WERNER. 1995. Interaction between food availability and predation mortality mediated by adaptive behavior. Ecology 76:2230-2234.

ANTONOVICS, J. 1968. Evolution in closely adjacent plant populations. VI. Manifold effects of gene flow. Heredity 23:507-524.

azevedo-Ramos, R. D., M. Van Sluys, J.-M. Hero, and W. E. MAGNUSSON. 1992. Influence of tadpole movement on predation by odonate naiads. J. Herpetol. 26:335-338.

Begon, M., J. L. Harper, and C. R. Townsend. 1996. Ecology. 3d ed. Blackwell Science, Oxford.

Berenbaum, M. R., A. R. Zangerl, and J. K. Nitao. 1986. Constraints on chemical coevolution: wild parsnips and the parsnip webworm. Evolution 40:1215-1228.

BERVEN, K. A. 1990. Factors affecting population fluctuations in larval and adult stages of the wood frog (Rana sylvatica). Ecology 71:1599-1608.

BLIGHT, A. R. 1976. Undulatory swimming with and without waves of contraction. Nature 264:352-354.

. 1977. The muscular control of vertebrate swimming movements. Biol. Rev. 52:181-218.

Brodie, E. D., A. J. Moore, AND F. J. JANZEN. 1995. Visualizing and quantifying natural selection. Trends Ecol. Evol. 10:315318.

Cook, S. A., AND M. P. Johnson. 1968. Adaptation to heterogeneous environments. 1 . Variation in heterophylly in Ranunculus flammula L. Evolution 22:496-516.

Dodson, S. I. 1989. Predator-induced reaction norms. BioScience 39:447-452.

DudLEY, S. A. 1996. Differing selection on plant physiological traits in response to environmental water availability: a test of adaptive hypotheses. Evolution 50:92-102.

Dudley, S. A., AND J. SChmitT. 1996. Testing the adaptive plas- 
ticity hypothesis: density-dependent selection on manipulated stem length in Impatiens capensis. Am. Nat. 147:445-465.

ENDLER, J. A. 1986. Natural selection in the wild. Princeton Univ. Press, Princeton, NJ.

FAlConer, D. S., AND T. F. C. MACKAY. 1996. Introduction to quantitative genetics. 4th ed. Longman, London.

FEDER, M. E. 1983. The relation of air-breathing and locomotion to predation on tadpoles, Rana berlandieri, by turtles. Phys. Zool. $56: 522-531$.

Folsom, T. C., AND N. C. Collins. 1984. The diet and foraging behavior of the larval dragonfly Anax junius (Aeshnidae), with an assessment of the role of refuges and prey activity. Oikos 42: $105-113$

FutUYMA, D. J., AND G. MORENO. 1988. The evolution of ecological specialization. Annu. Rev. Ecol. Syst. 19:207-233.

Futuyma, D. J., M. C. Keese, AND D. J. FunK. 1995. Genetic constraints on macroevolution: the evolution of host affiliation in the leaf beetle genus Ophraella. Evolution 49:797-809.

GoMULKIEWICZ, R., AND M. KIRKPATRICK. 1992. Quantitative genetics and the evolution of reaction norms. Evolution 46:390411.

HARVELl, C. D. 1986. The ecology and evolution of inducible defenses in a marine bryozoan: cues, costs, and consequences. Am. Nat. 128:810-823.

. 1990. The ecology and evolution of inducible defenses. Quart. Rev. Biol. 65:323-340.

HeDRICK, P. W. 1986. Genetic polymorphism in heterogeneous environments: ten years later. Annu. Rev. Ecol. Syst. 17:535-566.

JAIN, S. K., AND A. D. BRADShAW. 1966. Evolutionary divergence among adjacent plant populations. I. The evidence and its theoretical analysis. Heredity 21:407-441.

JoRDAN, C. E. 1992. A model of rapid-start swimming at intermediate reynolds number: undulatory locomotion in the chaetognath Sagitta elegans. J. Exp. Biol. 163:119-137.

KARBAN, R., AND J. H. MEYERS. 1989. Induced plant responses to herbivory. Annu. Rev. Ecol. Syst. 20:331-348.

KingSOLVER, J. G. 1995. Viability selection on seasonally polyphenic traits: wing melanin pattern in western white butterflies. Evolution 49:932-941.

LANDE, $R$. 1980. The genetic covariance between characters maintained by pleiotropic mutations. Genetics $94: 203-215$.

. 1984. The genetic correlation between characters maintained by selection, linkage, and inbreeding. Gen. Res. 44:309320.

LeVEne, H. 1953. Genetic equilibrium when more than one ecological niche is available. Am. Nat. 87:331-333.

Lewontin, R. C. 1957. The adaptation of populations to varying environments. Cold Spring Harbor Symp. Quant. Biol. 22:395408.

LIVELY, C. M. 1986a. Canalization versus developmental conversion in a spatially variable environment. Am. Nat. 128:561-572.

- 1986b. Competition, comparative life histories, and maintenance of shell dimorphism in a barnacle. Ecology 67:858-864.

- 1986c. Predator-induced shell dimorphism in the acorn barnacle Chthamalus anisopoma. Evolution 40:232-242.

McCollum, S. A., AND J. D. Leimberger. 1997. Predator-induced morphological changes in an amphibian: predation by dragonflies affects tadpole color, shape, and growth rate. Oecologia 109:615-621.

McCollum, S. A., AND J. VAN Buskirk. 1996. Costs and benefits of a predator-induced polyphenism in the gray treefrog Hyla chrysoscelis. Evolution 50:583-593.

MORAN, N. A. 1992. The evolutionary maintenance of alternative phenotypes. Am. Nat. 139:971-982.

Nodzenski, E., R. J. Wassersug, AND R. F. INGer. 1989. Developmental differences in visceral morphology of megophrynine pelobatid tadpoles in relation to their body form and model of life. Biol. J. Linn. Soc. 38:369-388.

Schlichting, C. 1986. The evolution of phenotypic plasticity in plants. Annu. Rev. Ecol. Syst. 17:667-693.

SChmitT, J., A. C. MCCormaC, AND H. SMiTh. 1995. A test of the adaptive plasticity hypothesis using transgenic and mutant plants disabled in phytochrome-mediated elongation responses to neighbors. Am. Nat. 146:937-953.

ScotT, D. E. 1994. The effect of larval density on adult demographic traits in Ambystoma opacum. Ecology 75:1383-1396.

Semlitsch, R. D., D. E. Scott, and J. H. K. PeChmann. 1988. Time and size at metamorphosis related to adult fitness in $\mathrm{Am}$ bystoma talpoideum. Ecology 69:184-192.

SHERIDAN, A. K., AND J. S. F. BARKER. 1974. Two-trait selection and the genetic correlation. II. Changes in the genetic correlation during two-trait selection. Aust J. Biol. Sci. 27:89-101.

Simms, E. L. 1992. Costs of plant resistance to herbivory. Pp. 392 425 in R. S. Fritz and E. L. Simms, eds. Plant resistance to herbivores and pathogens: ecology, evolution, and genetics. Univ. of Chicago Press, Chicago.

SKELLY, D. K. 1994. Activity level and the susceptibility of anuran larvae to predators. Anim. Behav. 47:465-468.

. 1995. A behavioral trade-off and its consequences for the distribution of Pseudacris treefrog larvae. Ecology 76:150-164. . 1996. Pond drying, predators, and the distribution of Pseudacris tadpoles. Copeia 1996:599-605.

Skelly, D. K, AND E. E. WeRnER. 1990. Behavioral and life historical responses of larval American toads to an odonate predator. Ecology 71:2313-2322.

SMITH, D. C. 1983. Factors controlling tadpole populations of the chorus frog (Pseudacris triseriata) at Isle Royale, Michigan. Ecology 64:501-510.

- 1987. Adult recruitment in chorus frogs: effects of size and date at metamorphosis. Ecology 68:344-350.

SmITH, D. C., AND J. VAN BUSKIRK. 1995. Phenotypic design, plasticity, and ecological performance in two tadpole species. Am. Nat. 145:211-233.

SPITZE, K. 1992. Predator-mediated plasticity of prey life history and morphology: Chaoborus americanus predation on Daphnia pulex. Am. Nat. 139:229-247.

Thompson, J. N. 1994. The coevolutionary process. Univ. of Chicago Press, Chicago.

$V_{I A}, S$. 1984. The quantitative genetics of polyphagy in an insect herbivore. I. Genotype-environment interaction in larval performance on different host species. Evolution 38:881-895.

-1991. The genetic structure of host plant adaptation in a spatial patchwork: demographic variability among reciprocally transplanted pea aphid clones. Evolution 45:827-852.

1994. The evolution of phenotypic plasticity: what do we really know? Pp. 35-57 in L. A. Real, ed. Ecological genetics. Princeton Univ. Press, Princeton, NJ.

VIA, S., AND R. LANDE. 1985. Genotype-environment interaction and the evolution of phenotypic plasticity. Evolution 39:505523.

WASSERSUG, R. J. 1989. Locomotion in amphibian larvae (or "Why aren't tadpoles built like fishes?"). Am. Zool. 29:65-84.

WASSERSUG, R. J., AND K. Hoff. 1979. A comparative study of the buccal pumping mechanism of tadpoles (Anura: Amphibia). Biol. J. Linn. Soc. 12:225-259.

1985. Kinematics of swimming in anuran larvae. J. Exp. Biol. 119:1-30.

Watkins, T. B. 1996. Predator-mediated selection on burst swimming performance in tadpoles of the pacific tree frog, Pseudacris regilla. Physol. Zool. 69:154-167.

WEBB, P. W. 1984. Body form, locomotion, and foraging in aquatic vertebrates. Am. Zool. 24:107-120.

WERNER, E. E., AND B. R. ANHOLT. 1993. Ecological consequences of the tradeoff between growth and mortality rates mediated by foraging activity. Am. Nat. 142:242-272.

- 1996. Predator-induced behavioral indirect effects: consequences to competitive interactions in anuran larvae. Ecology 77:157-169.

Wilbur, H. M. 1984. Complex life cycles and community organization in amphibians. Pp. 195-224 in P. W. Price, C. N. Slobodchikoff, and W. S. Gaud, eds. Wiley, New York.

WoODWARD, B. D. 1983. Predator-prey interactions and breedingpond use of temporary-pond species in a desert anuran community. Ecology 64:1549-1555.

Corresponding Editor: C. Lively 